\title{
Investigation of a national outbreak of STEC Escherichia coli O157 using online consumer panel control methods: Great Britain, October 2014
}

\author{
C. SINCLAIR ${ }^{1,2,3}$, C. JENKINS ${ }^{4}$, F. WARBURTON ${ }^{2}$, G. K. ADAK ${ }^{5}$ AND \\ J. P. HARRIS ${ }^{5,6 *}$ \\ ${ }^{1}$ UK Field Epidemiology Training Programme, Public Health England, London, UK \\ ${ }^{2}$ National Infection Services, Public Health England, London, UK \\ ${ }^{3}$ European Programme for Intervention Epidemiology Training (EPIET), European Centre for Disease \\ Prevention and Control, (ECDC), Stockholm, Sweden \\ ${ }_{5}^{4}$ Gastrointestinal Bacteria Reference Unit, National Infection Services, Public Health England, London, UK \\ ${ }^{5}$ Gastrointestinal Infections Department, National Infections Service, Public Health England, London, UK \\ ${ }^{6}$ NIHR Health Protection Research Unit in Gastrointestinal Infections, University of Liverpool, UK
}

Received 16 August 2016; Final revision 31 October 2016; Accepted 18 November 2016; first published online 14 December 2016

\section{SUMMARY}

In October 2014, Public Health England (PHE) identified cases of Shiga toxin-producing Escherichia coli (STEC) serogroup O157 sharing a multiple locus variable-number tandem repeat analysis (MLVA) profile. We conducted a case-control study using multivariable logistic regression to calculate adjusted odds ratios (aOR) and 95\% confidence intervals (CI) testing a range of exposures. Cases were defined as laboratory-confirmed STEC O157 with the implicated MLVA profile, were UK residents aged $\geqslant 18$ years with symptom onset between 25 September and 30 October 2014, and had no history of travel abroad within 5 days of symptom onset. One hundred and two cases were identified. Cases were mostly female $(65 \%$; median age 49 , range 2-92 years). It was the second largest outbreak seen in England, to date, and a case-control study was conducted using market research panel controls and online survey methods. These methods were instrumental in the rapid data collection and analysis necessary to allow traceback investigations for short shelf-life products. This is a new method of control recruitment and this is the first in which it was a standalone recruitment method. The case-control study suggested a strong association between consumption of a ready-to-eat food and disease (aOR 28, 95\% CI $5 \cdot 0-157$ ) from one retailer. No reactive microbiological testing of food items during the outbreak was possible due to the short shelf-life of the product. Collaboration with industrial bodies is needed to ensure timely traceback exercises to identify contamination events and initiate appropriate and focused microbiological testing and implement control measures.

Key words: Outbreak; panel control methods, salad, STEC O157.

\section{INTRODUCTION}

Shiga toxin-producing Escherichia coli (STEC) serogroup $\mathrm{O} 157$ belong to the Enterobacteriaceae family and are transmitted via the faecal-oral route [1]. The group is defined by its ability to produce Shiga toxin type 1 (Stx1) or Stx2 or both. Common exposures are

\footnotetext{
* Author for correspondence: Dr J. P. Harris, Farr Institute@HeRC, University of Liverpool, 2nd Floor, Block F, Waterhouse Buildings, 1-5 Brownlow Street, Liverpool, L69 3GL, UK. (Email: john.harris@liverpool.ac.uk)
} 
contaminated food, water and direct or indirect contact with animals or their environment. The infectious dose is low; symptoms range from mild diarrhoea, to bloody stools and more serious outcomes, including haemolytic uraemic syndrome (HUS) and death [2].

Between 2011 and 2015, reported cases of STEC O157 in England and Wales averaged $~ 800$ per year [3]. E. coli $\mathrm{O} 157$ isolated on selective agar at local hospital laboratories from faecal specimens from hospital or community patients with symptoms of gastrointestinal disease are submitted to the Gastrointestinal Bacterial Reference Unit (GBRU) for confirmation and typing. All isolates are phage-typed and prior to June 2015 when whole genome sequencing was implemented, molecular typing was performed on all isolates using multilocus variable number tandem repeat analysis (MLVA).

The majority of outbreaks reported between 1983 and 2012 were caused by phage type (PT) 21/28 $(34.9 \%)$ and PT8 (12.5\%) [4]. The largest outbreak in England was caused STEC O157 PT8 and was associated with the consumption of leeks and potatoes [5]. Other outbreaks in the UK have been associated with ready-to-eat packaged salad leaves or raw vegetables [6, 7], and a large outbreak of O104 in Germany and France was associated with consuming contaminated fenugreek seed sprouts $[8,9]$.

Ready-to-eat foods often have a short shelf-life making it difficult to investigate outbreaks where they are the likely vehicle of infection. By the time the first cases are confirmed and the outbreak is identified, the number of new symptomatic cases is often declining, and the food vehicle stocks diminished or exhausted, reducing the likelihood of detecting a pathogen in the food. Timely and comprehensive epidemiological and traceback investigations are therefore essential to provide robust evidence of the likely vehicle of infection, identify where in the production process a contamination event might have occurred and implement effective interventions.

In October 2014, Public Health England (PHE) identified 15 STEC O157 PT8 Stx1+2 isolates with the same MLVA profile [or a single locus variant (SLV) of that profile] within a 7-day period. The aim of this investigation is to identify the vehicle of infection, and to establish the extent of the outbreak using MLVA profiling and enhanced surveillance questionnaires (ESQ). This study also aimed to identify and implement control measures to prevent further infections occurring, both within this outbreak and in other foodborne outbreaks in the future.

\section{METHODS}

\section{Microbiological methods}

In England and Wales, local laboratories are responsible for culturing faecal specimens for E. coli O157. Presumptive isolates are then referred to the GBRU, PHE for confirmation and further typing. Scottish isolates are sent to the Scottish E. coli Reference Laboratory (SERL) in Edinburgh.

Detection and confirmation of STEC at GBRU included biochemical identification and serotyping of bacterial isolates. Real-time polymerase chain reaction (PCR) was performed to determine the presence of stxl and/or st $x 2$ [10]. Strains belonging to serogroup O157 were further differentiated by phage-typing and MLVA. Amplification of eight MLVA loci was performed in $20 \mu \mathrm{l}$ reaction volumes on all isolates in two quadruplex PCR reactions. VNTR-10 locus was not tested. The method was modified to use the following dye labels in the forward primers in the amplifications: NED in VNTR3 and VNTR17; 6-FAM in VNTR34, VNTR19, VNTR9 and VNTR36 and VIC in VNTR25 and VNTR37. Sizing of the amplified products was on an ABI 3730 Genetic Analyzer with 600 LIZ (Applied Biosystems, USA), size standard and data were analysed with Peakscanner software (Applied Biosystems). Fragment sizes were imported into Bionumerics software (bioMérieux, France) via an algorithm that calculated the tandem repeat numbers for each locus [11]. The outbreak strain was STEC O157 PT8 Stx1+2. All had the MLVA profile 17-8-15-5-4-3-6-6, or a SLV of that profile.

\section{Case ascertainment and descriptive epidemiology}

A case was described as a case of STEC O157 PT8 Stx $1+2$ as confirmed by GBRU or SERL with an onset of diarrhoea on or after 25 September 2014. Cases were required to be a resident of England, Scotland or Wales and to have been infected with the outbreak strain as defined by MLVA typing or a SLV thereof.

Descriptive information for the English cases was obtained from the enhanced surveillance scheme for STEC, described in detail elsewhere [12]. Briefly, the scheme requires all laboratories to submit isolates of STEC to the GBRU for confirmation and typing. Additionally all cases of STEC are contacted by their local Health Protection Team to obtain a standardized exposure questionnaire (the ESQ) and identify and follow-up contacts as part of routine case management. The questionnaire requests information 
about travel history, foods handled and purchased as well as animal contact; the questionnaire is available online (https://www.gov.uk/government/publications/ vero-cytotoxin-producing-escherichia-coli-questionnaire). Cases from Wales and Scotland were followed up through routine surveillance by their respective public health body and the information was sent to PHE for collation and analysis.

This initial data provided information on the age, sex, geographical distribution, clinical symptoms and severity of illness in cases, and food and environmental exposures.

\section{Hypothesis generation}

The first 15 cases were contacted in order to conduct hypothesis-generating questionnaires. This is a long and detailed questionnaire asking about various exposures including foods handled and consumed and if so any brands or types and where they were purchased, possible animal contact, and environmental exposures. These questionnaires are normally administered to the most recently identified cases. Some cases might already have completed the standardized enhanced surveillance questionnaire. The hypothesis-generating questionnaires were administered either by telephone or a web-based survey, depending on the responder's preference. Where exposures were reported by $70 \%$ of cases in the hypothesis-generating questionnaires they were used to inform the design of the case-control study questionnaire.

\section{Case-control study}

A case-control study was conducted to estimate the odds of exposure in cases compared to those in healthy controls for a range of exposures including, but not exclusively: the handling or consumption of potatoes, root vegetables, tomatoes, apples or bananas, identified in the hypothesis-generating questionnaires, in addition to commonly consumed foods (even if they fall below the $70 \%$ cut-off from the hypothesis-generating questionnaire) such as various meat products and salad vegetables. The outbreak case definition (described above) for the case-control study was further restricted to those aged $\geqslant 18$ years in order to ensure those being sent the electronic survey could complete it without the need for parental consent. Cases were excluded from the case-control study if they reported travel abroad in the 5 days prior to onset of illness.
Controls were recruited through a private company from a panel of registered participants. To register, participants must have been aged $\geqslant 18$ years. Both cases and controls were sent a link to an online survey using Select Survey web-based software. Due to software limitations, controls were not able to be frequency-matched to cases. Participants were limited to residents within the UK and any with a history of travel within the 5 days before completion were excluded. Recruitment of controls was curtailed at 100 individuals based on a 2:1 control:case ratio on cases applicable at the time of sending out the survey.

\section{Statistical analysis}

Initial univariable analysis, comparing exposures in cases and controls was carried out for each of the food types or where food was reported as being either handled or consumed and from where it was purchased.

Multivariable analysis was then conducted in order to find out which exposures were significantly and independently associated with being a case. This was undertaken in two stages. First, a backwards stepwise procedure was undertaken looking at all food types deemed to be significant, with an odds ratio $>1$ and a $P$ value $<0 \cdot 2$, from the univariable analysis. Second, for the food types that were found to be significant in this model (the first multivariable model), additional variables, such as handling, washing and retailer information were added into the second backwards stepwise analysis. For example, if somebody did not eat or handle potatoes, all the other potato variables were set to 'No' (because they would not have been purchased). Potatoes in a sack, not washing potatoes, home-grown potatoes and potatoes bought at other shops were not included due to the small number of cases and controls exposed. Both models were adjusted for age and sex.

\section{Additional epidemiological enquiries}

Following the results of the case-control study, all cases were asked additional follow-up questions via email. These were centred on the specific implicated food products. Approval was sought from cases for supermarkets to access their purchase history via loyalty card information and to provide any receipts they may have kept from purchases in the time before they became ill.

At each stage, the investigations were guided by the available epidemiological information. Initially, the approach involved discussions with the wider food industry to allow two-way sharing of information. As 


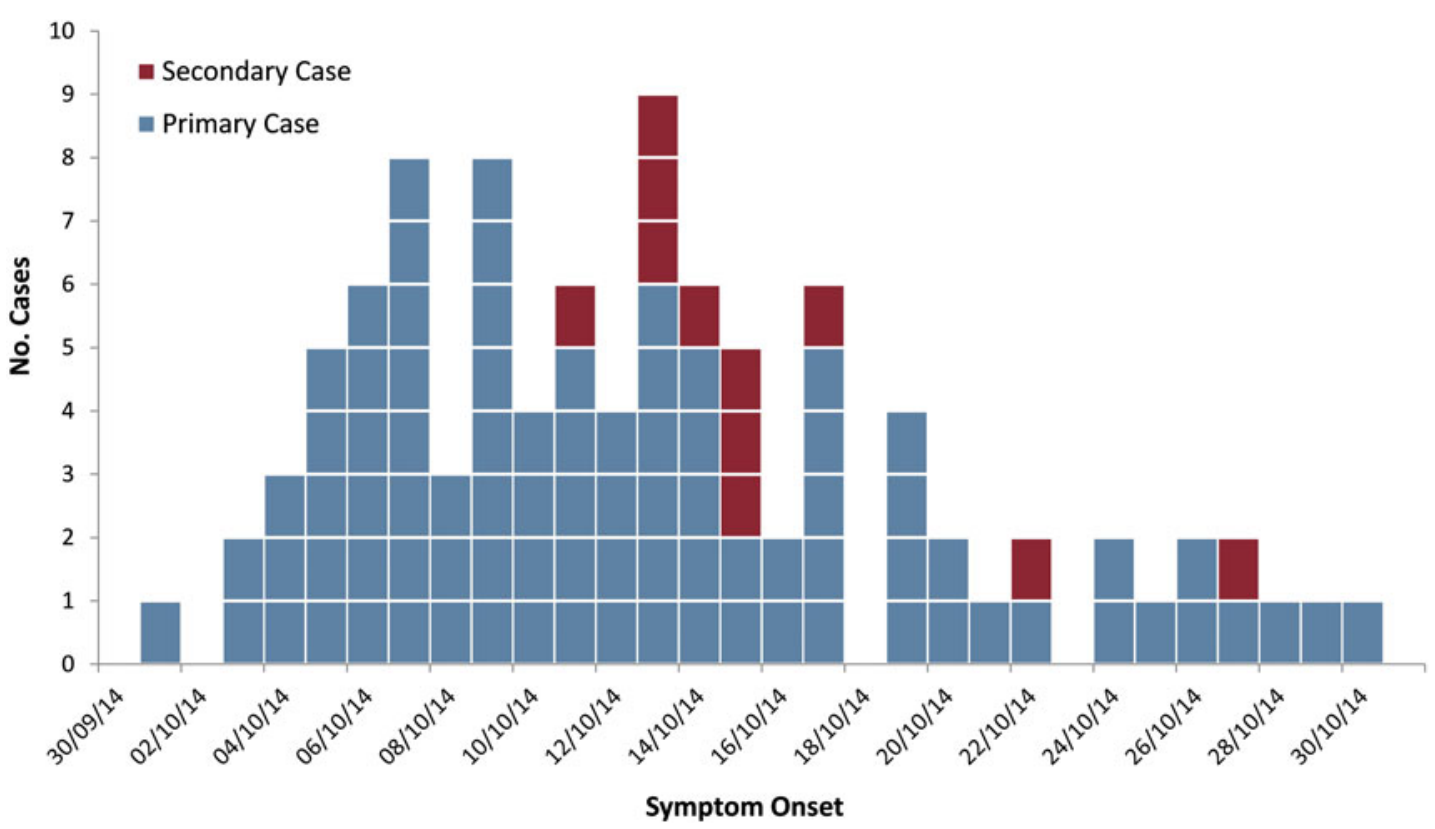

Fig. 1. Distribution of outbreak STEC cases by date of symptom onset, UK $(n=97)$.

the epidemiological findings progressed more detailed discussions were held with retailer no. 2. An estimated exposure period was provided by PHE and where available the cases' loyalty card numbers were also provided to retailer no. 2 with the aim of seeking specific purchase history information on foods available for consumption and within their shelf-life during the exposure period that may be relevant to the investigation. Retailer no. 2 provided details of relevant products, their suppliers, the stores that these products were supplied to and the name and country of the grower. Retailer no. 2 also provided a summary of the results of routine testing for indicator $E$. coli (i.e. non-pathogenic) in October from the relevant suppliers.

\section{RESULTS}

\section{Descriptive epidemiology}

A total of 102 cases of STEC PT8 Stx1 +2, with the outbreak MLVA profile were reported in England, Scotland and Wales with onset dates between 1 and 30 October 2014, with the peak of the outbreak occurring between 5 and 17 October (Fig. 1). Symptom information was available for 95 (93\%) of the 102 cases, with diarrhoea (100\%), abdominal pain (87.4\%) and blood in stool $(68.4 \%)$ being the most common symptoms. A high proportion of hospitalizations (33.7\%) were seen in cases, but there were none diagnosed with HUS and no deaths were associated with this outbreak.
Eighty-nine were defined as primary cases, 12 as secondary cases and in one case the status was unable to be established. Primary cases occurred in all regions within the UK, with the exception of Northern Ireland. The highest rates were reported in two regions; Wessex (4.1 cases $/ 1000000$ population) and Anglia \& Essex ( $2 \cdot 6$ cases $/ 1000000$ population).

The median age for primary cases was 49 years [interquartile range (IQR): 25-65]. Fifty-eight (65\%) cases were female. This compares to a median age of 27 (IQR 6-51) years and 50\% female in sporadic STEC cases reported in the same month.

\section{Analytical study}

Of the 89 confirmed, primary cases in England, Scotland and Wales, $77(86 \cdot 5 \%)$ were aged $\geqslant 18$ years and therefore eligible for inclusion in the case-control study. Fifty-six were successfully contacted and email addresses obtained for 53 cases, all of whom were emailed the survey. Of those emailed, the survey failed to deliver to three addresses and two participants replied to say they did not wish to complete the survey.

There were 43 responses to the case questionnaire, of which there were two pairs of duplicates, two were aged $<18$ years and one had a history of travel. Two responses were excluded as they were received after the cut-off date on 09:00 hours on Monday 17 November. The final response rate of those included in the study was $64 \%(36 / 56)$. 
Table 1. Odds ratios for univariable analysis of food types and were food was purchased (only displaying those with $O R>1$ and $P<0 \cdot 2$ )

\begin{tabular}{|c|c|c|c|c|c|c|c|}
\hline \multirow[b]{2}{*}{ Exposure } & \multicolumn{3}{|l|}{ Cases } & \multicolumn{3}{|c|}{ Controls } & \multirow[b]{2}{*}{ OR $(95 \% \mathrm{CI})$} \\
\hline & Total & Exposed & $\%$ & Total & Exposed & $\%$ & \\
\hline Eating mixed-leaf salad (raw) & 34 & 28 & $82 \cdot 4$ & 85 & 21 & $24 \cdot 7$ & $14 \cdot 2(5 \cdot 3-38 \cdot 1)$ \\
\hline Handling bananas & 36 & 29 & $80 \cdot 6$ & 96 & 44 & $45 \cdot 8$ & $4 \cdot 9(2 \cdot 0-12 \cdot 0)$ \\
\hline Handling tomatoes & 36 & 29 & $80 \cdot 6$ & 96 & 47 & 49 & $4 \cdot 3(1 \cdot 8-10 \cdot 6)$ \\
\hline Eating broccoli (cooked) & 29 & 18 & $62 \cdot 1$ & 82 & 29 & $35 \cdot 4$ & $3 \cdot 0(1 \cdot 3-7 \cdot 1)$ \\
\hline Handling broccoli & 36 & 19 & $52 \cdot 8$ & 96 & 29 & $30 \cdot 2$ & $2 \cdot 6(1 \cdot 2-5 \cdot 6)$ \\
\hline Eating carrots (raw) & 26 & 9 & $34 \cdot 6$ & 85 & 15 & $17 \cdot 6$ & $2 \cdot 5(0 \cdot 9-6 \cdot 5)$ \\
\hline Handling potatoes & 36 & 32 & $88 \cdot 9$ & 96 & 73 & 76 & $2 \cdot 5(0 \cdot 8-7 \cdot 5)$ \\
\hline Handing leeks & 36 & 6 & $16 \cdot 7$ & 96 & 7 & $7 \cdot 3$ & $2 \cdot 5(0 \cdot 8-7 \cdot 8)$ \\
\hline Handling carrots & 36 & 25 & $69 \cdot 4$ & 96 & 53 & $55 \cdot 2$ & $1 \cdot 8(0 \cdot 8-4 \cdot 1)$ \\
\hline Eating potatoes (cooked) & 33 & 28 & $84 \cdot 8$ & 83 & 61 & $73 \cdot 5$ & $2 \cdot 0(0 \cdot 7-5 \cdot 7)$ \\
\hline Eating spring onions (raw) & 24 & 5 & $20 \cdot 8$ & 84 & 9 & $10 \cdot 7$ & $2 \cdot 2(0 \cdot 7-7 \cdot 1)$ \\
\hline Retailer no. 1 & 36 & 9 & 25 & 96 & 4 & $4 \cdot 2$ & $7 \cdot 7(2 \cdot 3-25 \cdot 3)$ \\
\hline Retailer no. 2 & 36 & 21 & $58 \cdot 3$ & 96 & 26 & $27 \cdot 1$ & $3 \cdot 8(1 \cdot 7-8 \cdot 3)$ \\
\hline Home-grown & 36 & 8 & $22 \cdot 2$ & 96 & 10 & $10 \cdot 4$ & $2 \cdot 5(0 \cdot 9-6 \cdot 7)$ \\
\hline Retailer no. 3 & 36 & 7 & $19 \cdot 4$ & 96 & 9 & $9 \cdot 4$ & $2 \cdot 3(0 \cdot 8-6 \cdot 6)$ \\
\hline
\end{tabular}

OR, Odds ratio; CI, confidence interval.

One hundred control questionnaires were completed. Four reported foreign travel in the 5 days prior to completing the questionnaire leaving a total of 96 controls.

The results of the univariable analysis are shown in Table 1. Cases were significantly more likely to report exposure to consumption of raw mixed-leaf salad [odds ratio (OR) 14·2, 95\% confidence interval (CI) 5·3-38·1], handling bananas (OR $4 \cdot 9,95 \%$ CI $2 \cdot 0-12 \cdot 0$ ), handling tomatoes (OR 4.3, 95\% CI 1·8-10.6), eating cooked broccoli (OR 3·0, 95\% CI 1·7-7·1) and handling broccoli (OR $2 \cdot 6,95 \% \mathrm{CI} 1 \cdot 2-5 \cdot 6)$. Two retailers were also identified as being significant; retailer no. 1 (OR 7·8, 95\% CI 2·3-25·3) and retailer no. 2 (OR 3.8, 95\% CI 1.7-8.3); however, buying form retailer no. 1 only accounted for nine of the 36 cases included in the analysis.

The results of the two multivariable analysis models are shown in Table 2. Cases were significantly more likely to report eating mixed-leaf salad compared to

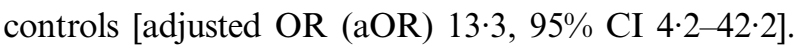
Adding in retailer information (model 2) suggests that cases were significantly more likely to have reported purchasing salad from retailer no. 2 (aOR 28.0, 95\% CI 5.0-157.4) and potatoes from retailer no. 2 (aOR $3 \cdot 3,95 \%$ CI $1 \cdot 0-10 \cdot 4)$ than controls.

\section{Further investigations}

Seventeen cases were able to provide more detailed information on the products they had consumed.
Nine (54\%) described consuming salad which contained baby leaves. Twelve $(71 \%)$ reported purchasing potatoes; however, there was no single brand and type of potato common to these cases. Additionally eight cases gave permission for the retailer to access their loyalty card information, and six provided receipts.

The Food Standards Agency carried out investigations on food chain information, microbiological analysis and purchase histories for a subset of the cases with retailer no. 2, alongside wider discussions with the food industry, as part of the outbreak investigation. Retailer no. 2 sold over 170 different bagged salad products. Following the analysis of more detailed food history information from PHE on a subset of 17 cases in relation to purchasing salad items from retailer no. 2's stores (i.e. type of product/type of packaging/area in which the store was located), provision of the estimated exposure period for the initial 52 primary cases calculated by PHE (28 September to 9 October 2014) and, where available, the cases' loyalty card numbers were also provided with the aim of seeking specific purchase history information regarding bagged salads, this was narrowed down to 37 products and three suppliers. Subsequently, filtering the list of products to show only those with 'baby leaf' in the title and exclude products in bowls, given that the cases specifically referred to purchasing baby leaf salad sold in bags. This left four bagged salad product lines produced by two suppliers. 
Table 2. Exposures in the final multivariable logistic regression analysis models, adjusted for age group and sex

\begin{tabular}{llll}
\hline \hline & Exposure & OR $(95 \% \mathrm{CI})$ & $P$ value \\
\hline Model 1 & Potatoes & $4 \cdot 1(0 \cdot 8-20 \cdot 5)$ & $0 \cdot 09$ \\
& Bananas & $3 \cdot 1(1 \cdot 0-9 \cdot 7)$ & $0 \cdot 06$ \\
Model 2 & Eating raw salad & $13 \cdot 3(4 \cdot 2-42 \cdot 2)$ & $<0 \cdot 001$ \\
& $\begin{array}{c}\text { Salad from } \\
\text { retailer no. 2 }\end{array}$ & $28 \cdot 0(5 \cdot 0-157 \cdot 4)$ & $<0 \cdot 001$ \\
& $\begin{array}{c}\text { Potatoes from } \\
\text { retailer no. 2 }\end{array}$ & $3 \cdot 3(1 \cdot 0-10 \cdot 4)$ & $0 \cdot 04$ \\
& & \\
\hline \hline
\end{tabular}

OR, Odds ratio; CI, confidence interval.

Retailer no. 2 indicated that there were no significant issues with routine testing results in September or October for the suppliers of the four products. A summary of the results of routine testing for indicator $E$. coli (i.e. non-pathogenic) in October from the relevant suppliers showed that indicator $E$. coli were detected at 390 colony-forming units (c.f.u.)/g in a product containing spinach. Retest samples had results of 250 and 180 c.f.u./g. There was no stock remaining for sale when retests were completed. Environmental testing was reviewed and all of the results were satisfactory. Follow-up test results for E. coli $\mathrm{O} 157$ and Salmonella were negative. Indicator $E$. coli were detected at 160 c.f.u./g in a second product line, which also contained spinach. Results of resampling were satisfactory. No further action was taken. The microbiological criteria for foodstuffs regulation (EC 2073/2005) includes a process hygiene criterion, which applies to all pre-cut and ready-to-eat fruits and vegetables including bagged salad, which states: 'Out of 5 samples, 2 can contain indicator E. coli between 100 c.f.u./g and 1000 c.f.u./g but that none of the five results should exceed 1000 c.f.u./g. The action in case of unsatisfactory results would include improvements in production hygiene and selection of raw materials.'

The results outlined above therefore comply with the maximum limit of 1000 c.f.u./g. Retailer no. 2 indicated that follow-up investigations had been conducted, following the detection of indicator E. coli results between 100 and 1000 c.f.u./g, and this has not resulted in the detection of pathogens. They were unable to identify any evidence that could shed light on a source of contamination of the products associated with the outbreak.

\section{DISCUSSION}

The investigation of foodborne outbreaks associated with STEC O157 can be challenging as they are usually small in case numbers and geographically dispersed. These characteristics are indicative of low-level, intermittent contamination of nationally distributed food products. The time delay between exposure and follow-up interviews inevitably reduces accurate patient recall. Furthermore, outbreaks associated with the consumption of contaminated salad leaves can be especially challenging as they are often served as a side dish or minor component of a meal that people are unlikely to remember [7].

The proactive use of a highly discriminatory molecular-typing method (e.g. MLVA) on all isolates of STEC O157 submitted to GBRU facilitated the identification of this outbreak [11]. PT8 is the second most common phage type in the UK and the salad exposure linking the cases epidemiologically was not immediately apparent from the initial analysis of the ESQ, so it would have been difficult to identify and confirm a link between cases without the moleculartyping data.

This was the second largest outbreak of STEC O157 investigated by PHE comprising of 102 cases, with a $64 \%$ response rate for the case-control study of those who were contactable. This was a good response rate for an outbreak investigation and probably reflects the severity of illness, and therefore people's interest in identifying the cause. This outbreak presented a good opportunity to assess the impact of the epidemiological and traceback investigations.

Bagged salad leaves (pre-packaged types) were not implicated in the analysis in either the hypothesis generating questionnaires or ESQs. Identifying possible exposures at the hypothesis generating stage is a matter of pragmatism. The rule of thumb of including foods that trigger $70 \%$ is also tempered by other considerations. For example, if cheese is reported by over $70 \%$ of respondents but analysis of the types of cheese suggest that they are varied this is considered as an unlikely culprit. Alternatively if a food that is not normally consumed in high levels or something very specific is mentioned then this might be an indication and would be included further in the investigation. In this investigation detailed questions on consumption of mixed-leaf salad products were not initially collected (specifying bagged or pre-packaged salads) during the case-control study. Therefore, follow-up questions had to be circulated to all cases after initial 
analysis had been completed. This hindered the investigation and the delay may have had an impact on case recall, as people are often unable to recall their food history over a long period of time. Furthermore, there is evidence of Respondent and/or Interviewer bias towards meat products as the most likely source of STEC O157 infection and details of the specific product line of bagged salad, and the salad leaf content, are often missing from the ESQ.

While mixed-leaf salad products containing baby leaves were implicated, due to the short shelf-life of salad products, it was not possible to obtain samples for reactive microbiological testing of the suspected food items in response to the outbreak. Routine microbiological testing results for STEC O157 supplied by retailer no. 2 were negative and so we were unable to obtain any microbiological results to confirm the epidemiological evidence. However, testing of foods often does not lead to the isolation of STEC as, due to the low infectious dose, levels of bacteria below the detection limits of the tests are sufficient to cause disease [13].

Identification of the most likely contaminated food vehicle can be delayed by problems in obtaining suitable control subjects for case-control investigations using traditional methods. This is due to the fact that many households in the UK are dispensing with landlines and there are no directories of mobile numbers and the increased use of call screening and caller identifying display to prevent cold calls. The use of market research panel control subjects via online surveys during the investigation described in this study meant that the epidemiological data for the casecontrol study was collected quickly, with 100 control subjects being contacted in less than 1 week. This is far quicker than could have been achieved by a more traditional telephone method, and was less costly in terms of manpower effort. Additionally, as the questionnaires were completed online, there was minimal data cleaning. This allowed for analysis to be carried out and results to be presented to the Outbreak Control Team within 2 days of the survey being closed. The food vehicle was identified quickly, which allowed timely food traceback investigations to be initiated. The cost for this service [£3.20 sterling ( €3.70) per questionnaire] was far less than carrying out telephone interviews when taking into consideration the hours which would be needed to recruit the controls, making market research panels a rapid and cost-effective control recruitment method.

A limitation of the analytical study was differences in the age and sex profiles between the cases and controls. It is possible that this may have introduced some selection bias into the study and therefore the controls may not necessarily be reflective of the population from which the cases are drawn. However, we were able to adjust for age and sex in the multivariable analysis; neither were significant in the model. In future surveys it will be possible to recruit controls based upon the age and sex distribution of cases in order to minimize this limitation.

Market research panel controls is a relatively new method of recruiting controls. This study was the second within PHE to utilize this approach. The first study was part of an investigation into an outbreak of Salmonella Mikawasima, and compared the panel control method with a traditional control recruitment method [14]. The authors found that the panel control method was a quicker and more cost-effective method of control recruitment and recommended this approach for outbreak investigations in order to reduce recall bias. However, like this study there were some differences in the demographics of the controls compared to the cases.

Due to an increasing number of outbreaks involving short shelf-life products, it is necessary to investigate and identify these products as quickly as possible. This study found the use of market research panel controls and online surveys to be a cost-effective investigation method which allowed the study to be conducted in a timely manner. However, it is necessary to develop this methodology further to ensure that controls are representative of the case population. More detailed standardised online survey templates for hypothesis generation questionnaires, including details of the specific product line of bagged salad and the salad leaf content are required in order to ensure the rapid follow up of cases prior to the analytical study.

In light of the difficulties in detecting STEC O157 in contaminated salad and raw vegetables, resources should be focused on identifying the location of the growers of the most likely contaminated food vehicle, assessing the biosecurity at the implicated farms and testing animal faecal samples in the farm environment.

Heightening the awareness of these outbreaks is important for both public health authorities and industry, in order to stimulate analysis of events that can lead to contamination. A multi-organizational approach, involving governmental and industrial bodies must be taken to ensure adequate routine batch testing of products by the food business operator and that trace back exercises and appropriate and focused microbiological testing can be carried out promptly once a vehicle has been identified. 


\section{ACKNOWLEDGEMENTS}

The authors acknowledge all members of the Outbreak Control Team for their role in the outbreak investigation. We particularly acknowledge Lynda Browning and Eleanor Anderson of Public Health Scotland, Chris Williams and Rob Smith of Public Health Wales for providing data on their cases. We also thank Marie Anne Chattaway, Neil Perry and Vivienne dos Nascimento at GBRU for their microbiological expertise. Drazenka Tubin-Delic and the Food Standards Agency for conducting the traceback exercise, and finally, Lisa Byrne, Kirsten Glen and Natalie Adams from the GI Department, PHE.

John Harris is affiliated to the National Institute for Health Research Health Protection Research Unit (NIHR HPRU) in Gastrointestinal Infections at University of Liverpool in partnership with Public Health England (PHE), in collaboration with University of East Anglia, University of Oxford and the Institute of Food Research. John Harris is based at the University of Liverpool. The views expressed are those of the author(s) and not necessarily those of the NHS, the NIHR, the Department of Health or Public Health England.

\section{DECLARATION OF INTEREST}

None.

\section{REFERENCES}

1. Heymann DL. Control of Communicable Diseases Manual, 20th edn. APHA Press, 2014.

2. Byrne L, et al. The epidemiology, microbiology and clinical impact of Shiga toxin-producing Escherichia coli in England, 2009-2012. Epidemiology \& Infection 2015; 143: 3475-3487.

3. McCormick J, et al. Report of annual human infection data for 2015. Epidemiology of Foodborne Infections Group, 2016.
4. Adams NL, et al. Shiga toxin-producing Escherichia coli O157, England and Wales, 1983-2012. Emerging Infectious Diseases 2016; 22: 590-597.

5. Launders $\mathbf{N}$, et al. A large Great Britain-wide outbreak of STEC O157 phage type 8 linked to handling of raw leeks and potatoes. Epidemiology \& Infection 2016; 144: $171-181$.

6. Launders N, et al. Outbreak of Shiga toxin-producing E. coli O157 associated with consumption of watercress, United Kingdom, August to September 2013. Eurosurveillance 2013; 18.

7. Byrne L, et al. Epidemiological and microbiological investigation of an outbreak of severe disease from shiga toxin-producing Escherichia coli O157 infection associated with consumption of a slaw garnish. Journal of Food Protection 2016; 79: 1161-1168.

8. King LA, et al. Outbreak of Shiga toxin-producing Escherichia coli O104:H4 associated with organic fenugreek sprouts, France, June 2011. Clinical Infectious Diseases 2012; 54: 1588-1594.

9. Buchholz U, et al. German outbreak of Escherichia coli O104:H4 Associated with sprouts. New England Journal of Medicine 2011; 365: 1763-1770.

10. Jenkins C, et al. Assessment of a real-time PCR for the detection and characterization of verocytotoxigenic Escherichia coli. Journal of Medical Microbiology 2012; 61: 1082-1085.

11. Byrne L, et al. Evaluating the use of multilocus variable number tandem repeat analysis of Shiga toxin-producing Escherichia coli $\mathrm{O} 157$ as a routine public health tool in England. PLoS ONE 2014; 9: e85901.

12. Launders $\mathbf{N}$, et al. Disease severity of Shiga toxinproducing E. coli $\mathrm{O} 157$ and factors influencing the development of typical haemolytic uraemic syndrome: a retrospective cohort study, 2009-2012. BMJ Open 2016; 6 .

13. Jenkins C, et al. Public health investigation of two outbreaks of shiga toxin-producing Escherichia coli O157 associated with consumption of watercress. Applied and Environmental Microbiology 2015; 81: 3946-3952.

14. Mook P, et al. Selection of population controls for a Salmonella case-control study in the UK using a market research panel and web-survey provides time and resource savings. Epidemiology \& Infection 2016; 144: $1220-1230$. 